\title{
Book Review_Diagnostic pathology: head and neck, 2nd edition
}

\author{
Alena Skálová 1 \\ Received: 16 July 2016 / Accepted: 17 July 2016/Published online: 8 August 2016 \\ (C) Springer-Verlag Berlin Heidelberg 2016
}

The second edition of Diagnostic Pathology: Head and Neck, edited by Drs. Thompson, Wenig, Muller, and Nelson has recently been published. Part of the highly appreciated Diagnostic Pathology series, this updated volume is an invaluable diagnostic aid for every practicing pathologist and resident interested in head and neck pathology.

The second edition of Diagnostic Pathology: Head and Neck has more than 1100 pages organized into 10 sections, including the nasal cavity and paranasal sinuses, pharynx, larynx and trachea, oral cavity, salivary glands, jaw, ear and temporal bone, neck, thyroid gland, and parathyroid glands. Each section includes a short introduction with a short overview of anatomy and histology of the site, followed by nonneoplastic lesions, benign, and malignant tumors (total of 1120 different entities). More than 4300 illustrations and graphics are included in this book and in the accompanying electronic version, highlighting diagnostic features for each entity.

I was pleased to see that the most common problematic topics in head and neck pathology are addressed objectively. Many newly recognized and poorly understood entities are included, such as mammary analog secretory carcinoma of salivary glands, HPV-associated oropharyngeal carcinoma, EWSR1-driven tumors, molecular pathways as targets for salivary duct carcinoma, cribriform adenocarcinoma of minor salivary glands, and much more. High-quality, carefully annotated color images provide clinically and diagnostically

Alena Skálová

skalova@fnplzen.cz

1 Charles University in Prague, Faculty of Medicine in Plzen, Czech Republic important information on more than 315 new and evolving entities of the head and neck and endocrine organs.

This second edition incorporates the most recent clinical, pathological, histological, and molecular knowledge in the field to provide a comprehensive overview of all key issues relevant to today's practice, covering all aspects of head and neck pathology.

The key facts and outstanding images, including gross and microscopic features, a wide range of stains, and detailed illustrations make this book an extremely useful tool in diagnostic practice. Fully integrated, searchable, and linked content between differential diagnostic categories is perfectly suited for residents, while updated genetic testing algorithms, new images, and outstanding graphics make this text ideal for both residents and practitioners. With the well-structured layout, subjects are easy to find, and the relevant information is presented in brief terms. The e-book version online makes finding what you are looking for even easier and comes with additional images of superb quality that support key points made in the text.

In summary, the second edition of Diagnostic Pathology: Head and Neck provides one of the top resources for pathologists with an interest in head and neck lesions. Ease of use in daily diagnostic practice, the quality of the information, and the support of excellent quality and well-chosen images will contribute to its success. The bulleted format makes the book user friendly in a busy practice.

Highly recommended!

Lester D. R. Thompson and Bruce M. Wenig (Eds.)

Print ISBN 9780323392556

$1192 \mathrm{pp}$

$£ 211.99$

http://www.elsevierhealth.co.uk/diagnostic-pathology-headand-neck-9780323392556.html 